This manuscript is a reproduction of the paper "Implementation of K-band Mushroom meta-material filter for satellite applications" by A. Arsanjani, L. Robins, R. Teschl, W. Bosch, originally published in the Proceedings of the 50th European Microwave Conf., Utrecht, The Netherlands, Jan. 2021, pp. 495-498. The copyright owner is the European Microwave Association, EuMA.

\title{
Implementation of K-band Mushroom meta-material filter for satellite applications
}

\author{
Arash Arsanjani ${ }^{\# 1}$, Luke Robins ${ }^{\# 2}$, Reinhard Teschl${ }^{\# 3}$, Wolfgang Bösch ${ }^{\# 4}$ \\ \#Institute of Microwave and Photonic Engineering, Technische Universität Graz, Graz, Austria \\ $\left\{{ }^{1}\right.$ arash.arsanjani, ${ }^{2}$ lrobins, ${ }^{3}$ reinhard.teschl, ${ }^{4}$ wbosch $\} @$ tugraz.at
}

\begin{abstract}
This paper presents a novel metamaterial structure which achieves high performance in terms of loss, power handling and size reduction compared to traditional waveguides. The design procedure and considerations have been elaborated for additive manufacturing of the structure. The filter chosen to implement was a 4th-order filter with four poles; $400 \mathrm{MHz}$ bandwidth and a return loss of better than $15 \mathrm{~dB}$, at the center frequency of $18.5 \mathrm{GHz}$.
\end{abstract}

Keywords - Metamaterial, additive manufacturing, low loss, microwave filters.

\section{INTRODUCTION}

One of the primary drivers of the satellite communications industry is the demand for low-loss and high power technologies of components such as filters and antennas [1]. Conventional waveguides have wide adoption as they combine high power handling capability with low losses. This comes at the cost of the integration complexity, size and weight, which is of major concern in applications with strict limits and high costs associated with launch mass.

Planar technologies, on the other hand, address the issue by providing smaller size and simplified integration capability with other active and passive circuits. These advantages, as well as ease of manufacture and lower costs compared to waveguide technologies, meant planar structures saw widespread adoption in most applications over the past few decades. Substrate integrated waveguides (SIW) [2] and grounded co-planar waveguides (GCPW) have been developed and have improved radiation loss compared to micro-strips.

Modern communication systems have the added strain of rapidly increasing requirements of bandwidth and number of connections. To meet this growth, the exploitation of ever-higher frequency bands is necessary. Traditional planar technologies are increasingly unable to meet these requirements as the losses increase to unacceptable levels with frequency and are therefore limited to lower frequency applications, e.g. X-band. This includes SIW and GCPW structures, which still suffer from dielectrics losses at higher frequencies. These challenges have necessitated new technologies for use at these higher frequency bands while still maintaining a compromise of low loss, small size, ease of integration, and cost.

A method to mitigate the dielectric losses mentioned above is to completely remove the dielectric material. Following this method, air-filled SIW(AFSIW) structures were introduced in [3] in which the dielectric has been cut out from the waveguides to reduce the losses. AFSIW has the additional benefit of having higher average power handling capabilities when compared to the dielectric-filled SIWs (DFSIW) [3], [4]. Removing the dielectric comes with the cost of increased size as the field is in air. For example the structure proposed in [5] provides better loss performance but exhibits a size increase. This necessitates the development of miniaturisation techniques to try to keep the advantages of both types of structures.

A miniaturization technique based on metamaterial with high permittivity has been introduced in [6]. The basic principle of artificial magnetic conductor (AMC) makes use of resonant cavities formed between a periodic array and the ground plane [7]. This can be exploited for a limited frequency band such an AMC acts almost like a perfect magnetic conductor (PMC) and fully reflects incident waves with zero phase shift. So far, the AMC concept has mainly been used for low-profile antennas [8]. However, when the structure is used below the resonance frequency, new applications are possible. This concept was implemented as a multi-layer planar structure [6], which is capable of artificially increasing the relative dielectric permittivity of the used substrate. The technique was shown to enable exceptional size reduction capabilities at the expense of increased dielectric losses. This work proposes innovative techniques to remove the dielectric substrates of this miniaturization technique.

In this paper, a novel implementation of the mushroom metamaterial is proposed. The new structure exhibits better performance in terms of loss, power handling and size; which will be discussed. Additionally, different aspects of fabrication and challenges has been surveyed and discussed in the following sections. The structure's geometry makes the implementation by traditional manufacturing techniques challenging, necessitating exploring the use of additive manufacturing. Metal additive manufacturing (AM) has introduced innovative solutions for the fabrication of complicated microwave components [9]. As the technology is still developing, there can be issues of accuracy and reliability of this technique for the implementation of microwave components. AM also experiences issues with the conductivation, surface roughness, geometry optimization, limiting assembly with the fabrication of microwave components.

The paper is organized as below: the theoretical consideration of the design including unit-cell, resonator and 
This manuscript is a reproduction of the paper "Implementation of K-band Mushroom meta-material filter for satellite applications" by A. Arsanjani, L. Robins, R. Teschl, W. Bosch, originally published in the Proceedings of the 50th European Microwave Conf., Utrecht, The Netherlands, Jan. 2021, pp. 495-498. The copyright owner is the European Microwave Association, EuMA.

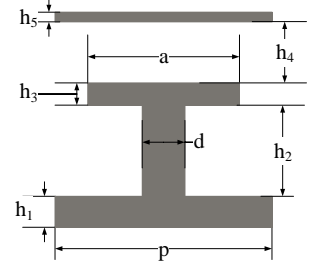

(a)

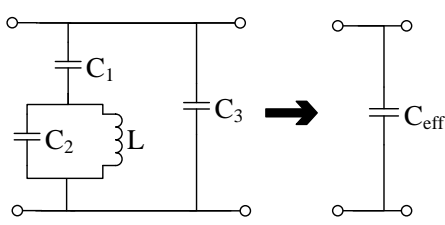

(b)
Fig. 1. Meta-material unit-cell structure (a) unit-cell model (b) equivalent circuit elements. $p=1.43 \mathrm{~mm}, a=1.23 \mathrm{~mm}, d=0.6 \mathrm{~mm}, h_{2}=0.5 \mathrm{~mm}$, $h_{3}=0.2 \mathrm{~mm}, h_{4}=0.23 \mathrm{~mm}$ (c) dispersion diagram of the proposed unit-cell; the highlighted frequencies is the targeted frequencies.

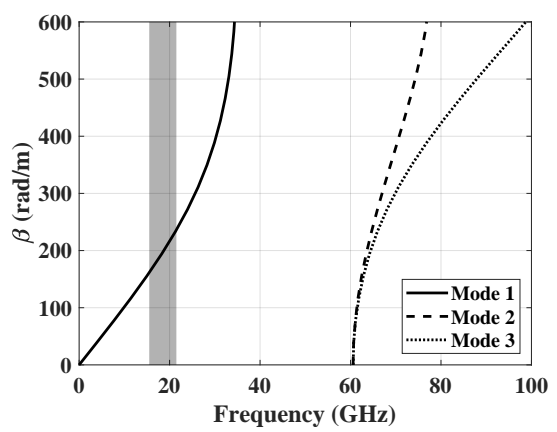

Fig. 2. Dispersion diagram of the proposed unit-cell; the highlighted frequencies are the targeted frequencies.

filter design are discussed in section II. The techniques and challenges of implementing the structure using current additive manufacturing is discussed in Section III. Section IV provides the simulation results; and section $\mathrm{V}$ will present a brief conclusion.

\section{TheORETICAL CONSIDERATIONS}

\section{A. Metamaterial substrate}

The first step begins with the design of the meta-material substrate, a unit cell of which is shown in Fig.1a. It was demonstrated before in [6] that the proposed unit-cell can be described by equivalent circuit elements. The equivalent circuit element is shown in Fig.1b. A general equation for $C_{\text {eff }}$ can be derived from the equivalent circuit elements, if the ohmic losses are ignored. The equivalent capacitor $C_{\text {eff }}$ as stated in [6] is obtained as follows:

$$
C_{\text {eff }}=C_{1} \frac{1-\omega^{2} L C_{2}}{1-\omega^{2} L\left(C_{1}+C_{2}\right)}+C_{3} .
$$

From the derivation in [6], the final equation for the equivalent $\varepsilon_{r_{\text {eff }}}$ is as follows:

$$
\varepsilon_{r_{\text {eff }}}(\omega)=\varepsilon_{r}\left(\frac{h_{2}+h_{3}+h_{4}}{h_{4}} \frac{a^{2}}{p^{2}} \alpha+\frac{p^{2}-a^{2}}{p^{2}}\right)
$$

where $\alpha$ is the dispersion factor and described as:

$$
\alpha=\frac{1-\left(\frac{\omega}{\omega_{p}}\right)^{2}}{1-\left(\frac{\omega}{\omega_{r}}\right)^{2}}
$$

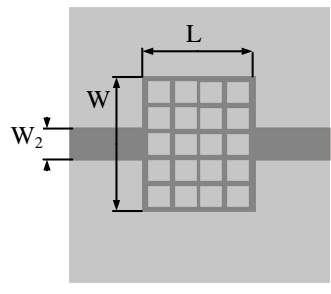

(a)

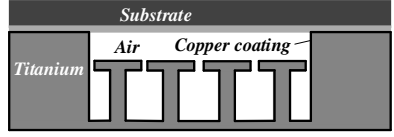

(b)
Fig. 3. Mushroom meta-material structure single resonator (a) top view; $L=$ $5.72 \mathrm{~mm}, W=7.15 \mathrm{~mm}, W_{2}=1.86 \mathrm{~mm}(\mathrm{~b})$ side view

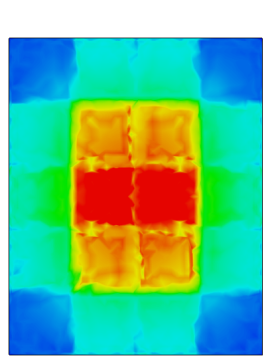

(a)

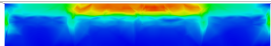

(c)

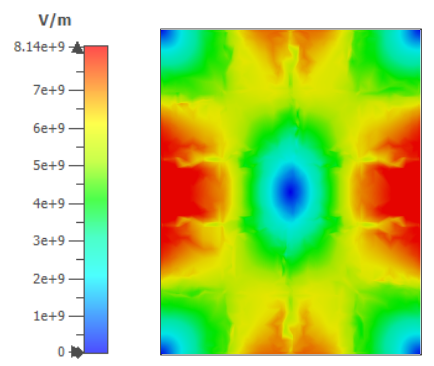

(b)

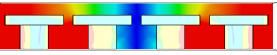

(d)
Fig. 4. Field distribution of a single resonator; (a) \& (c) electrical field distribution, (b) \& (d) magnetic field distribution.

$\omega_{r}=1 / \sqrt{L\left(C_{1}+C_{2}\right)}$ and $\omega_{p}=1 / \sqrt{L C_{2}}$ are the resonance and plasmon frequency, respectively [6].

Equation (2) demonstrates that the ratio between $\left(h_{2}+h_{3}+\right.$ $\left.h_{4}\right)$ and $h_{4}$ can be used to manipulate the effective dielectric constant, which can be used for miniturisation of the structure.

The dimensions of the unit-cell structure are determined by the required filter specifications which has a center frequency at $18.5 \mathrm{GHz}$, a bandwidth of $400 \mathrm{MHz}$ and a return loss of better than $15 \mathrm{~dB}$ in the pass-band. They must also be chosen with care to avoid propagation of other non desired modes in the unit-cell. Using eigenmode simulations, a dispersion diagram can be set up, which can be used to select the desired mode and region of operation. Fig. 2 demonstrates the first three mode propagation constant inside the unit-cell. The highlighted region shows the frequency spectrum which will be used for the implementation of the filter. It can be seen that the dispersion is approximately linear in this area.

\section{B. Resonator design}

Each resonator of the proposed filter is realised by a cavity loaded with a matrix of $4 \times 5$ functional metamaterial elements as depicted in Fig. 3a and Fig. 3b. For the dimensions given in Fig. 1a, using Equation 2, the effective dielectric permittivity of the airgap is calculated to be equal to $\varepsilon_{r_{\text {eff }}} \simeq 2.8$. The structure will consist of two parts, the bottom layer which is intended to be fabricated through additive manufacturing, and the top layer for which a metal sheet or simple substrate with 
This manuscript is a reproduction of the paper "Implementation of K-band Mushroom meta-material filter for satellite applications" by A. Arsanjani, L. Robins, R. Teschl, W. Bosch, originally published in the Proceedings of the 50th European Microwave Conf., Utrecht, The Netherlands, Jan. 2021, pp. 495-498. The copyright owner is the European Microwave Association, EuMA.

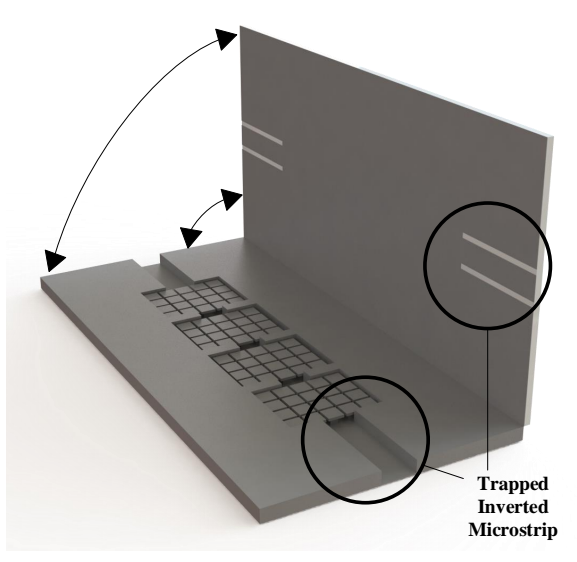

(a)

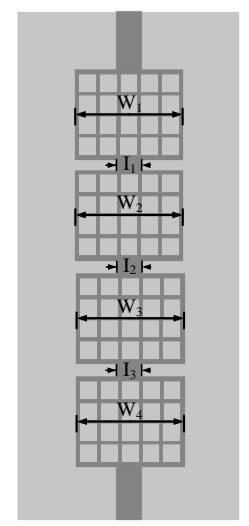

(b)

Fig. 5. (a) Proposed 4-th order filter with iris direct coupling (b) Top view of the proposed filter $W_{1}=W_{4}=7.32 \mathrm{~mm}, W_{2}=W_{3}=7.65 \mathrm{~mm}$, $I_{1}=I_{3}=2.34 \mathrm{~mm}, I_{2}=2.18 \mathrm{~mm}$.

conductive metal can be used. The use of a substrate for the top layer will allow the integration of tuning elements in the future. Optimal assembly of the complete component will be investigated.

The electric and magnetic field distributions of the first resonating mode inside the cavity are shown in Fig. 4. The electromagnetic field distribution resembles the first resonating mode inside a dielectric cavity and can be considered to be equivalent. The resonant frequency can be calculated using the $\varepsilon_{r_{\text {eff }}}$ of the metamaterial structure inside an equally sized dielectric-filled cavity resonator.

The size reduction can be easily calculated as the guided-wavelength inside a cavity is related to $\sqrt{1 / \varepsilon_{r_{\text {eff }}}}$, therefore it can be concluded that the size reduction in comparison to a air-filled resonator is calculated by the following equation:

$$
\text { Size reduction } \%=\frac{\varepsilon_{\mathrm{r}_{\text {eff }}}-1}{\varepsilon_{\mathrm{r}_{\text {eff }}}} \times 100 .
$$

Using the equation above, the size reduction is equal to $64 \%$ compared to an air-filled resonator.

\section{Filter design}

The proposed 4th-order filter is designed with direct coupled resonators, which is shown in Fig. 5a. The coupling between resonators is achieved using iris coupling as depicted in Fig. 5b. The irises shift the resonance frequency of each resonator, this can be compensated for each cavity separately by adjusting the width of each resonator, this can be done without redesigning the unit-cells. The input and output are coupled through trapped inverted microstrips (TIM)[10] as transitions to the first and last resonators. The $N+2$ coupling matrix which satisfies the filter specification in the linear configuration is given below:

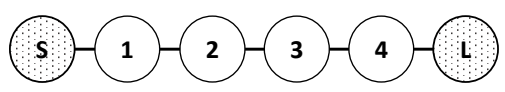

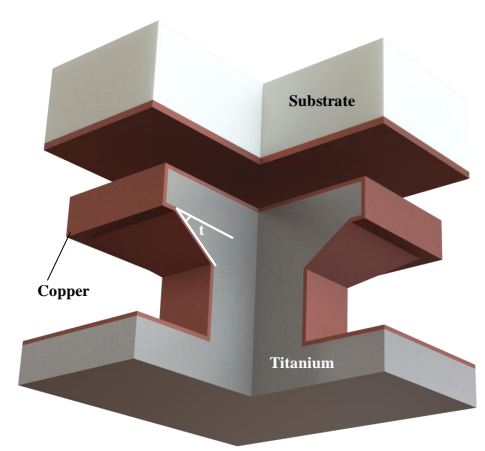

Fig. 6. Unitcell of the proposed mushroom with modified pins for AM, $t$ is the chamfer angle of the pin and is equal to $35^{\circ}$.

$$
M=\left[\begin{array}{cccccc}
0 & 0.91 & 0 & 0 & 0 & 0 \\
0.91 & 0 & 0.80 & 0 & 0 & 0 \\
0 & 0.80 & 0 & 0.64 & 0 & 0 \\
0 & 0 & 0.64 & 0 & 0.80 & 0 \\
0 & 0 & 0 & 0.80 & 0 & 0.91 \\
0 & 0 & 0 & 0 & 0.91 & 0
\end{array}\right]
$$

Average power handling capability (APHC) is determined by the temperature rise of the copper and titanium and the substrate [4]. Therefore, since the dielectric has been removed from the proposed structure a better performance is expected in comparison to dielectric filled structures.

\section{AdDitive MANUfACTURING IMPLEMENTATION}

In the introduction some of the challenges for AM were briefly outlined, the following section will further elaborate on these considerations as well as the design verification and modification to ensure the desired performance. The type of AM technology, machine and material choices can have very large effects on the tolerances and achievable results.

The envisaged printer to manufacture the structure makes use of direct metal laser sintering (DMLS) to manufacture metal parts, which welds the surfaces of metal particles together using a laser to create the CAD model. The achievable accuracy of the component depends on the spot size, layer height of the machine and particle size of the material used. The printer has a minimum layer thickness of $30 \mu \mathrm{m}$ and $100 \mu \mathrm{m}$ spot size when using titanium $(63 \mu \mathrm{m}$ max particle size). These tolerances were taken into consideration during the design stage. Additionally the titanium requires heat treatment once manufactured, which can cause stresses and shrinkage of the component. This sets a maximum overhang limitation on the structure of $100 \mu \mathrm{m}$ when moving from the vertical pins to the horizontal tops. To mitigate this, the corners between the pin and tops were chamfered, as depicted in Fig. 6. The chamfering led to a resonant frequency shift which was compensated for by adjusting the $h_{4}$ height.

The titanium base used has low conductivity and so requires coating with an additional more conductive copper layer. The coating to be used has a skin depth of $0.4862 \mu \mathrm{m}$ at the desired frequency, therefore to ensure the structure 
This manuscript is a reproduction of the paper "Implementation of K-band Mushroom meta-material filter for satellite applications" by A. Arsanjani, L. Robins, R. Teschl, W. Bosch, originally published in the Proceedings of the 50th European Microwave Conf., Utrecht, The Netherlands, Jan. 2021, pp. 495-498. The copyright owner is the European Microwave Association, EuMA.

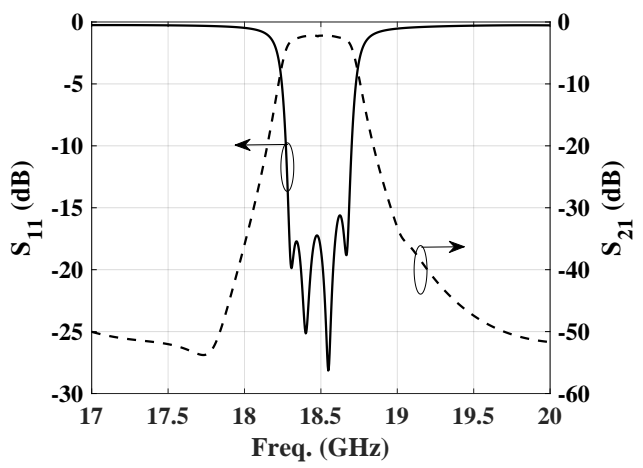

Fig. 7. Simulation results of 4-th order filter with fractional bandwidth of $3.25 \%$ and unloaded quality factor of 650

performs as designed, the coating is required to have a minimum thickness of $2 \mu \mathrm{m}$ as safety margin. The coating will be done using physical vapor deposition (PVD) and once complete will need to be electropolished to reduce the roughness and loss.

\section{RESULTS}

A 4th-order microwave filter with the bandwidth of 400 $\mathrm{MHz}$ and center frequency of $18.5 \mathrm{GHz}$ is simulated using CST; and the frequency response of the filter is reported in Fig. 7. The simulated filter shows an insertion loss of better than $2.5 \mathrm{~dB}$. By enhancing the new mushroom meta-material structure a size reduction of $64 \%$ compared to an air-filled structure, has been achieved which is in accordance with the analytical model of the meta-material structure. To highlight the advantages of the design, the quality factor of the filter has been simulated using CST for comparison with commercial low loss dielectric substrates. The prototype structure was designed to be fabricated with titanium and coated with copper with an electrical conductivity of $5.96 \times 10^{7} \mathrm{~S} / \mathrm{m}$, the simulation results show a quality factor of 600 .

Fig. 8a shows the quality factor of the metamaterial resonator as a function of the conductivity and Fig. 8b shows the quality factor for a dielectric-filled resonator with relative dielectric permittivity as a function of the loss tangent. A comparison highlights that to achieve similar performance one requires a very low loss tangent. Currently the only commercial substrate that can compete with the proposed structure is RO5880, with a loss tangent of 0.0009 and a dielectric permittivity of 2.2. Even though one can achieve a similar quality factor, the lower dielectric permittivity of the RO5880 leads to a larger size compared to the proposed metamaterial structure. It is worth highlighting that the dimensions of the prototype structure were selected carefully with the available AM technology in mind and this limits the achievable performance. Advances in AM technologies would make it possible for future structures to achieve higher performance.

\section{CONCLUSION}

This paper presents an implementation of a mushroom meta-substrate structure to improve the loss and size of

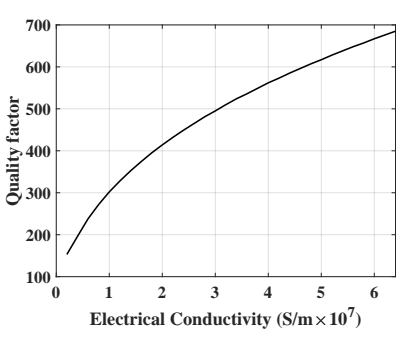

(a)

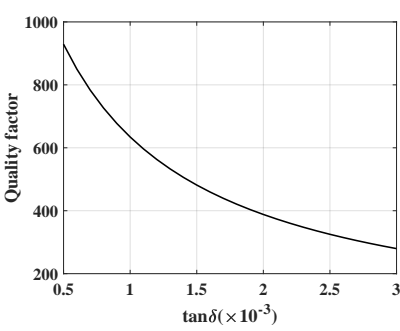

(b)
Fig. 8. (a) Quality factor of the metamaterial resonator for different electrical conductivity of conductors (b) Quality factor of the dielectric-filled resonator for different loss tangents.

microwave components. The proposed structure shows better performance compared to the good quality dielectrics on the market.

\section{ACKNOWLEDGEMENT}

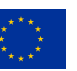
"This project has received funding from the European Union's Horizon 2020 research and innovation programme under the Marie Skłodowska-Curie grant agreement No 811232”.

\section{REFERENCES}

[1] G. Maral, M. Bousquet, and Z. Sun, Satellite communications systems: systems, techniques and technology. John Wiley \& Sons, 2020.

[2] M. Bozzi, A. Georgiadis, and K. Wu, "Review of substrate-integrated waveguide circuits and antennas," IET Microwaves, Antennas \& Propagation, vol. 5, no. 8, pp. 909-920, 2011.

[3] F. Parment, A. Ghiotto, T.-P. Vuong, J.-M. Duchamp, and K. Wu, "Air-filled substrate integrated waveguide for low-loss and high power-handling millimeter-wave substrate integrated circuits," IEEE Transactions on Microwave Theory and Techniques, vol. 63, no. 4, pp. 1228-1238, 2015.

[4] Y. J. Cheng, K. Wu, and W. Hong, "Power handling capability of substrate integrated waveguide interconnects and related transmission line systems," IEEE Transactions on Advanced Packaging, vol. 31, no. 4, pp. 900-909, 2008.

[5] N. H. Nguyen, F. Parment, A. Ghiotto, K. Wu, and T. P. Vuong, "A fifth-order air-filled siw filter for future $5 \mathrm{~g}$ applications," in 2017 IEEE MTT-S International Microwave Workshop Series on Advanced Materials and Processes for RF and THz Applications (IMWS-AMP). IEEE, 2017, pp. 1-3.

[6] X. Xu and J. Wei, "Miniaturisation design of patch antenna using a low-profile mushroom type meta-substrate tailored with high permittivity," IET Microwaves, Antennas \& Propagation, vol. 12, no. 7, pp. 1216-1221, 2018

[7] D. C. Li, F. Boone, M. Bozzi, L. Perregrini, and K. Wu, "Concept of virtual electric/magnetic walls and its realization with artificial magnetic conductor technique," IEEE microwave and wireless components letters, vol. 18 , no. 11 , pp. 743-745, 2008.

[8] A. P. Feresidis, G. Goussetis, S. Wang, and J. C. Vardaxoglou, "Artificial magnetic conductor surfaces and their application to low-profile high-gain planar antennas," IEEE Transactions on Antennas and Propagation, vol. 53, no. 1, pp. 209-215, 2005.

[9] O. A. Peverini, G. Addamo, M. Lumia, G. Virone, F. Calignano, M. Lorusso, and D. Manfredi, "Additive manufacturing of ku/k-band waveguide filters: A comparative analysis among selective-laser melting and stereo-lithography," IET Microwaves, Antennas \& Propagation, vol. 11, no. 14, pp. 1936-1942, 2017.

[10] T. C. Edwards and M. B. Steer, Foundations for microstrip circuit design. John Wiley \& Sons, 2016. 